UNIVERSITAT DE BARCELONA

FACULTAT DE MATEMÀTIQUES

\title{
SOME REMARKS ON HEYTING MATRICES
}

by

Josep M. Font

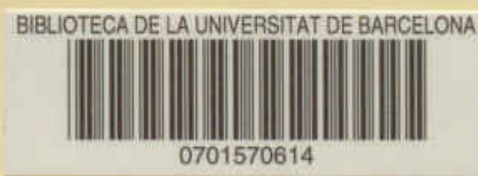

PRE-PRINT N. 039

Febrer 1986 

SOME REMARKS ON HEYTING MATRICES

by Josep M. Font

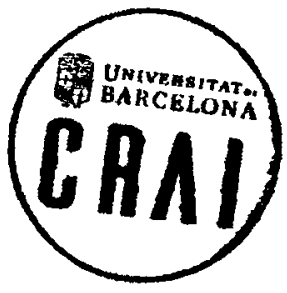

In the excellent book of W.A. Pogorzelski and P. Wojtylak "Elements of the theory of completeness in propositional logic" [ PW] the class of Heyting algebras is used to define a semantic consequence operator in the algebra of the propositional formulas. This operator is of course equal to the usual (axiomatic) syntatic consequence. The (rather methodological) purpose of this note is to point out that this semantic operator is exactly the classical one obtained using Heyting algebras as matrices in the ordinary way, and to explain why we think the latter approach is, in a sense, more natural

than the former. We shall follow the terminology and notations of [Pw] quite closely, with a few exceptions.

Throughout this paper we shall denote by $\mathcal{A}=\langle A, \div, \cup, \cap,-\rangle$ any algebra of type $(2,2,2,1)$ and by $S=\langle S,+,+, \cdot, \sim\rangle$ the algebra of propositional formulas constructed in the usual way from a set At of propositional variables; recall that any mapping $v: A t \rightarrow A$ can be extended in a unique way to an homomorphism $h^{v}: S \longrightarrow \mathcal{A}$. Now let $\mathcal{A}$ be a Heyting algebra, and for every $B \subseteq A$ let us denote by $F(B)$ the least (implicative) filter of $\mathcal{A}$ containing $B$. Then in page 79 of [PW] the consequence operation determined by 98 is defined as follows; for each $\alpha \in S$ and $X \subseteq S$ :

Accepted for publication in: Zeitschrift fur Mathematische Logik und Grundlagen der Mathematik. 
These opngequence operators are used in pages 83 and 84 to define

hoper filter consequence operation:

(2) $\alpha \in \underline{F C}(\mathrm{X})$ iff $a \in \vec{A}(\mathrm{X})$ for every Heyting algebra $\mathcal{A}$.

This is thought of as a semantic consequence operator, and in page 85 a completeness theorem is stated in the form $\operatorname{Ded}(X)=\underline{F C}(X)$ where we use $\operatorname{Ded}(X)$ instead of $\operatorname{Cn}\left(R_{0}, S b\left(A_{1}\right) \cup X\right)$, the syntactic consequences of $x$.

This semantic approach derives from the application of some very general concepts of q-algebras to the particular case of Heyting algebras. However, Heyting algebras can be also viewed as logical matrices, and so there is a more classical semantic approach to intuitionistic propositional logic still using Heyting algebras. We recall that a logical matrix is a pair $\mathrm{mt}=\left\langle\mathcal{A}, \mathrm{A}^{*}\right\rangle$ where $\mathcal{A}$ is an algebra and $A^{*} \subseteq A$ is the set of "distinguished elements". Then the matrix consequence associated with $m$ is (page 61 of [ $\mathrm{PW}]$ ):

(3) $\alpha \in \vec{m}(x)$ iff $h^{v}(x) \subseteq A^{*}$ implies $h^{v}(\alpha) \in A^{*}$ for every $v: A t \rightarrow A$

Any Heyting algebra can become a logical matrix by taking any filter as the set of disguished elements; however, as we shall see at the end of the note, it is enough to consider the case of the least filter. So we put forward the following

(4) Definitions: If $\mathcal{A}$ is a Heyting algebra then the matrix associated with $\mathcal{A}$ is $m[\mathcal{A}]=\langle\mathcal{A},(1)\rangle$ where 1 is the unit of $\mathscr{A}$. 
Then according to (3) the matrix consequence associated with $A$ is $\overrightarrow{m[A}](x)=\left\{\alpha \in S: h^{v}(x) \subseteq\{1\}\right.$ implies $h^{v}(\alpha)=1$ for every v: At $\longrightarrow$ A \}

(5) Proposition: If $H$ is a filter of a Heyting algebra $\mathcal{A}$, then $\overrightarrow{m[h]} \leq \overrightarrow{m[H / H]}$.

Proof: If is quite similar to the proof of Lemma 2.68 of [PW], its essential point being the use of the axiom of choice to find an embedding of $\mathscr{A} / \mathrm{H}$ into $\mathcal{A}$.

(6) Theorem: $\mathcal{A}=\overrightarrow{m[\mathscr{A}]}$ for every Heyting algebra $\mathcal{A}$.

Proof: Let $a \in S$ and $x \subseteq S$ be such that $\alpha \in \dot{\mathcal{A}}(x)$ and suppose that $v: A t \rightarrow A$ is such that $h^{v}(x) \subseteq\{1\}$. Then $h^{v}(\alpha) \in F\left(h^{v}(x)\right)=$ $=F(\{1\})=\{1\}$, that is $h^{v}(\alpha)=1$. Therefore $\alpha \in \overrightarrow{m[\mathcal{A}]}(x)$.

Conversely suppose that $\alpha \notin \vec{A}(y):$ then there is a $v: A t \rightarrow A$ such that $h^{v}(\alpha) \notin F\left(h^{v}(x)\right)$ and by a well-known property of Heyting algebras there is a prime filter $H$ of $\mathcal{A}$ such that $h^{v}(\alpha) \notin H$ and $F\left(h^{v}(x)\right) \subseteq H$. Now putting $w=p \circ v$ we find a $w: A t \longrightarrow A / H$ such that $h^{w}(a) \neq[1]$ but $h^{w}(x) \subseteq\{[1]\}$, that is, we have proved that $\alpha \notin \overrightarrow{m[\mathcal{A} / H]}(x)$. But by (5) this implies that $a \notin m[A](x)$.

So we see that the consequence operator of [PW] actually is the same as the matrix one, taking the unit as the only distinguished element. In the rest of the paper we see that this is what one gets in using any logical matrix, provided it is "good enough" for 
the intuitionistic propositional calculus. We shall split this requirement in two properties, each of a different character. We name the first one after $[D]$ :

(7) Definition: A logical natrix $m$ is said to be faithful (to the intuitionistic propositional calculus) iff Ded $\leq \vec{m}$.

(y) Lemma: A matrix $m=\left\langle\mathcal{A}, A^{*}\right\rangle$ is faithful if and only if it satisfies the two conditions:

a) $h^{v}(\alpha) \in A^{*}$ for every $v: A t \longrightarrow A$ and every theorem $\alpha$ of the intuitionistic propositional calculus; and

b) $A^{*}$ is closed under the application of Modus Ponens (that is, if $a \in A^{*}$ and $a \stackrel{\rightarrow}{\rightarrow} b \in A^{*}$ then $b \in A^{*}$, for each $\left.a, b \in A\right)$.

Proof: Quite trivial. For the "if" part, use the Compactness and the Deduction Theorems for Ded.

The second requirement is that the matrix does not show "unnecessary" differences between its elements. It has been used since the appearance of the earliest studies of logical matrices in the thirties (for instance, from [LT] and [I] through [NT] to [L], and related works of these and other authors), though mixed with other properties and under different names, such as "regular", "normal", and so on. We have singled out it and given it one of these names:

(9) Definition: A logical matrix $m=\left\langle\mathcal{A}, A^{*}\right\rangle$ is said to be regular Iff for each $a, b \in A$, if $a \dot{\rightarrow} b \in A^{*}$ and $b \dot{\rightarrow} a \in A^{*}$ then $a=b$. 
(10) Theorem: let $n t=\left\langle\mathscr{A}, A^{*}\right\rangle$ be a faithful logical matrix. Then:

(a) The relation $R$ on $A$ defined by: $a R b$ iff $a \rightarrow b \in A^{*}$ and $b \doteqdot a \in A^{*}, \quad$ is a congruence relation of $\mathcal{A}$;

(b) $A^{*}$ constitutes a single equivalence class under $R ;$ and (c) The "quotient matrix" $m 1 / R=\left\langle\mathcal{A} / R, A^{*} / R\right\rangle$ is faithrul and regular, and $\vec{m}=\overrightarrow{m / R}$.

Proof: (a) Given several elements of the matrix $a, b, c \in A$ we can always define a valuation $v: A t \rightarrow A$ such that $h^{v}(\alpha)=a, h^{v}(B)=$ $=b$ and $h^{v}(\gamma)=c$ for some $\alpha, \beta, \gamma \in S$; taking into account that $m$ is faithful and Lemma 8 , we see that $A^{*}$ includes all elements of A with the algebraic "form" of a theorem of the intuitionistic calculus. Now the theorems $a+\alpha$ and $(a+\beta)+((\beta+\gamma)+(a+\gamma))$ imply that $R$ is an equivalence relation, and, with $(\alpha+\beta)+((Y+\alpha) \rightarrow(\gamma+\beta))$, that it is a congruence with respect to $\dot{\rightarrow}$. The theorems $a \cdot \beta \rightarrow \alpha$, $\alpha \cdot \beta+\beta$ and $(\gamma+\alpha)+((\gamma+\beta)+(\gamma+\alpha \cdot \beta))$ make the trick for $n$, their duals (for + ) do the same for $U$, and the case of - requires only $(\alpha+\beta)+(\sim \beta \rightarrow \sim \alpha)$.

(b) If $a R b$, we have $a \div b \in A^{*}$, so from $a \in A^{*}$ we get $b \in A^{*}$ by applying Modus Ponens; if $a, b \in A^{*}$ then the theorems $a+(B \rightarrow \alpha)$ and $\beta+(\alpha+B)$ imply that $b \stackrel{\rightarrow}{a} \in A^{*}$ and $a \dot{\rightarrow} b \in A^{*}$, that is, a R b. Therefore $A^{*}$ constitutes exactly one equivalence class.

(c) Part (b) in particular talls us that $R$ is a "congruence of the matrix" $m$ and then it is known (see the proof in [ $w$ ], for instance) that the quotient matrix $m / R=\left\langle 4 / R, A^{*} / R\right\rangle$ is equivalent (as a matrix) to $m$, that is, that $\vec{m}=\overrightarrow{m / R}$. Therefore, $m / R$ is also faithful. Finally, in the quotient $[a] \rightarrow[b] \in A^{*} / R$ iff 
iff $a \stackrel{\dot{\rightarrow}}{b} \in A^{*}$, so the fact that $A^{*}$ constitutes a single equivalence class implies that $I / / R$ is regular.

Now we can see why in the case of Heyting algebras we restricted us, in (4), to $A^{*}=\{1\}$. If $H$ is a filter of a Heyting algebra $\mathcal{A}$, then $\langle\mathscr{A}, \mathrm{H}\rangle$ is a faithful matrix, the congruence defined in $10(\mathrm{a})$ is the congruence associated with $H$, and therefore the quotient matrix is $\langle\mathcal{A} / \mathrm{H},[\mathrm{H}]\rangle$. But $[\mathrm{H}]=[[1]\}$ is the unit of $\mathcal{A} / \mathrm{H}$, that is, the "general" matrix $\langle\mathcal{A}, \mathrm{H}\rangle$ is equivalent, by theorem 10 , to a matrix of the type chosen in definition (4). In our last result we see that this class of matrices is the class of all quotients obtained in the preceding theorem.

(i1) Theorem: Let $m=\left\langle\mathscr{A}, A^{*}\right\rangle$ be a logical matrix. Then $m$ is faithful and regular if and only if $\mathcal{A}$ is a Heyting algebra and $A^{*}=\{1\}$ where 1 is the unit of $\mathcal{A}$.

Proof: Suppose $m$ is faithful and regular. Then $1(b)$ tells us that

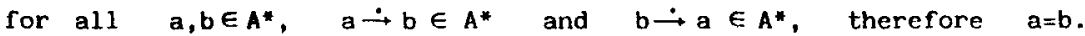
That is, $A^{*}$ has only one element, say 1 . Now we define a binary relation on $A$ : $a \leq b$ if $a \dot{\rightarrow} b=1$. Using the same techniques of theorem 12 and the same theorems quoted there, it is easy to see that $\leq$ is an order relation on $A$ and 1 is 1 ts maximum, and moreover that $\langle A, \cap, U\rangle$ is a lattice. The theorem $(\alpha+(\beta \rightarrow \gamma)) \rightarrow(\alpha \cdot \beta+\gamma)$ implies that fir each $a, b, c \in A, a \leq b \dot{\rightarrow}$ iff $a \cap b \leq c$, that is, $\mathcal{A}$ is a relatively pseudo-complemented (or implicative) lattice. Then from $a+(\sim a+B)$ we find that $a n-a=b n-b=0$ for $a l 1 \quad a, b \in A$, and so there is a minimum 0 of $\mathcal{A}$. Finally the theorems 
$\sim \alpha \rightarrow(\alpha+\alpha \cdot \sim \alpha)$ and $(\alpha \rightarrow \alpha \cdot \sim \alpha) \rightarrow \sim \alpha$ allow us to see that $\sim \alpha$ is deducl

ble from $\alpha+\alpha^{*} \sim \alpha$ and conversely, therefore in $\mathcal{A} \quad-a=a \rightarrow 0$ for

all $a \in A$. That is, we conclude that $\mathcal{A}$ is a Heyting algebra and 1 is its unit.

For the converse part of the theorem, it is well-known that any Heyting algebra with its unit is a faithrul matrix, and it is indeed regular because $a \stackrel{\circ}{b}=b \stackrel{b}{a}=1$ implies $a=b$.

(12.) Corollary. A logical matrix $m$ is faithful if and only if there is a Heyting algebra $\mathcal{A}$ such that $\vec{m}=\overrightarrow{m[M]}$.

Proof: If in is faithful then theorem 10 implies that $\vec{m}=\overrightarrow{\| 1 / R}$ and that $m / R$ is faithful and regular, therefore by theorem $11, A / R$ is a Heyting algebra and $A^{*} / R=\{1\}$ where 1 is the unit of $\mathscr{A} / R$. In other words, $m / R=m[\mathcal{A} / R]$ and so $\vec{m}=\overrightarrow{m[A / R}]$. The converse is trivial.

This corollary sufficiently expresses our conclusion: Heyting matrices are "all" faithful matrices; taking theorem 6 into account we see that from them we can obtain the algebraic semantics of [PW] for the intuitionistic propositional calculus. We have presented detailed proofs for this restricted case because our interest in these remarks was mainly methodological. However a large number of calculi can be treated in a similar way, and we must say the theory of abstracts logics of Brown, Suszko and others (see [BS] and related works) provides a general framework for a lot of cases with several extremely general results. It is rather surprising that this 
powerful approach has been neglected in [PW].

The author wishes to express his thanks to Dr. V. Verdú for his valuable comments on the general subject and contents of this note.

\section{References}

[BS] D.J. Brown - R. Suszko "Abstract logics" Dissertationes Mathematicae CII (1973) 9-41.

[D] M. Dummett "Elements of Intuitionism" Clarendon Press, Oxford, 1977.

[L] E.J. Lemmon "An extension algebra and the modal system $T$ " Notre Dame Journal of Formal Logic 1 (1960) 3-12.

[LT] J. bukasiewicz - A. Tarski "Untersuchungen uber den Aussagenkalkül" Comptes rendus des séances de la Société des Sciences et des lettres de Varsovie 23 (1930) cl III, 30-50.

[MT] J.C.C. McKinsey - A. Taraki "Some theorems about the sentential calculi of Lewis and Heyting" The Journal of Symbolic Logic 13 (1948) 1-15.

[PW] W.A. Pogorzelski - P. Wojtylak "Elements of the theory of completeness in propositional logic" The Silesian University, Katowice, 1982.

[ T] A. Tarski "Der Aussagenkalkül und die Topologie" Fundamenta Mathematicae 31 (1938) 103-134.

[W] R. Wójcicki "Matrix approach in the methodology of sentential calculi" Studia Logica 32 (1973) 7-39.

Postal address: Josep M. Font

Faculty of Ma shematics

University of Barcelona

Gran Via 585, 08007 Barcelona, Spain 



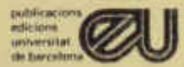

Dipòsit Legal B.: 4.534-1986

BARCELONA - 1986 